\title{
ARROW-MANGASARIAN SUFFICIENT CONDITIONS FOR CONTROLLED SEMIMARTINGALES*
}

\author{
Nils Chr. Framstad ${ }^{\dagger}$
}

\begin{abstract}
This paper shows a version of Arrow's generalization of Mangasarian's sufficient conditions valid for controlled stochastic differential equations driven by semimartingales. The infinite case is covered. An example is given.
\end{abstract}

Key words: Optimal control, semimartingales, sufficient maximum principle. MSC (2000): 93E20, 49K45, 60G48, 60H10, $91 \mathrm{~B} 28$.

\section{Introduction}

This paper gives a verification theorem for optimality of a controlled stochastic differential equation with semimartingale driving noise. We employ a version of Arrow's generalization of Mangasarian's conditions (the sufficiency version of Pontryagin's maximum principle) known from deterministic control theory, first proved rigorously by Seierstad and Sydsæter in [12].

Various necessity and sufficiency results are known in Markovian framework; references include Kushner [8], Bismut [3], Bensoussan [2], Haussmann [6], Peng [10], Cadenillas and Haussmann [4] for necessary conditions for controlled diffusions, while Tang and Li [14] provide the same in a jump diffusion setting; see also Kabanov [7]. Sufficient conditions for controlled jump diffusions are given by Framstad, Øksendal and Sulem [5]. This paper is an extension to the semimartingale case. The generalization turns out to be straightforward, as the product rule for differentiation works similarly; as for the transversality conditions, they are as in the deterministic case.

What is not equally straightforward, is applying the theory. The result involves backward stochastic differential equations (BSDEs). While the major research effort in the theory for BSDEs has been dedicated to the Brownian or Wiener-Poisson Markov case, an early paper by Antonelli [1] treats the semimartingale setting.

The outline of the paper is as follows: Section 1 gives the basic result, which is just what we would expect from the Markovian set-up; in Section 2 we shall see why the usual generalizations apply. Finally, Section 3 gives an example with an application to portfolio selection.

\footnotetext{
${ }^{*}$ Supported by the Research Council of Norway. The paper has benefited from discussions with Bernt $\varnothing \mathrm{ksendal}$ and Yaozhong $\mathrm{Hu}$.

${ }^{\dagger}$ Dept. of Mathematics, University of Oslo, Box 1053 Blindern, N-0316 Oslo, Norway. ncf@math.uio.no
} 


\section{The main result}

Assume given a filtered probability space $\left(\Omega, \mathfrak{S},\left\{\mathcal{F}_{t}\right\}_{t \geq 0}, \mathrm{P}\right)$ satisfying the usual conditions. Our controlled system will be driven by an increasing process $A$, by an integer-valued random measure $M$ without fixed jump times and by an $\mathbf{R}^{n}$-valued continuous local martingale $Z=\left(Z_{1}, \ldots, Z_{n}\right)^{\top}$ where, throughout the paper, the superscript ¿ $^{\top}{ }^{\top}$ denotes matrix transpose. We assume that the cross-variation matrix of $Z$ and the predictable measure-valued process $\Pi$ associated to $M$ are absolutely continuous with respect to $\mathrm{d} A$, such that for a suitable $\beta:[0, T] \rightarrow \mathbf{R}^{n \times n}$ with components $\beta_{i j}$ and a suitable measure $\lambda$, one has

$$
\mathrm{d}\left[Z_{i}, Z_{j}\right]=\beta_{i j} \mathrm{~d} A, \quad \Pi(\mathrm{d} t, \mathrm{~d} z)=\lambda(\mathrm{d} z) \mathrm{d} A(t) .
$$

We assume that the $\mathbf{R}^{d}$-valued state variable $X$ obeys the stochastic differential equation

$$
\mathrm{d} X(t)=b(t, X(t), u(t)) \mathrm{d} A(t)+\sum_{i=1}^{n} \sigma_{i}(t, X(t), u(t))^{\top} \mathrm{d} Z_{i}(t)+\int \eta\left(t, X\left(t^{-}\right), u(t), z\right) \bar{M}(\mathrm{~d} t, \mathrm{~d} z)
$$

where $\bar{M}$ is «compensated at small jumps», i.e. for a suitable truncation function $\chi$ which may be taken to have values in $\{0,1\}, \mathrm{d} \bar{M}=\mathrm{d} M-\chi(z) \lambda(\mathrm{d} z) \mathrm{d} A(t)$. In (2), both $b$ and the $\sigma$ are given measurable functions $[0, T] \times \mathbf{R}^{d} \times U \rightarrow \mathbf{R}^{d}$, while $\eta$ is a given measurable function $[0, T] \times \mathbf{R}^{d} \times U \times \Xi \rightarrow \mathbf{R}^{d}$ for some mark space $\Xi$. The given set $U$ is called the control region and we are free to choose the value of $u(t, \omega)$ from $U$, subject to the requirement that $u$ is a predictable process and gives rise to a unique strong solution $X(t)=X^{(u)}(t)$ of $(2)$ on the time interval $t \in[0, T]$. Such controls are called admissible if in addition (5) below holds, and also we have the following $\mathrm{L}^{2}$ condition:

$$
\mathrm{E}\left[\int_{0}^{T}\left\{\sum_{i, j=1}^{n}\left(\sigma_{i}^{\top} \sigma_{j}\right)(t, X(t), u(t)) \beta_{i j}+\int\left(\eta^{\top} \eta\right)\left(t, X\left(t^{-}\right), u(t), z\right) \chi(z) \lambda(\mathrm{d} z)\right\} \mathrm{d} A(t)\right]<\infty
$$

ensuring that the integrals with respect to the local martingales (i.e. $Z$ and the «compensated small jumps» part) indeed have zero mean. A controlled process $X=X^{(u)}$ satisfying (2) on $[0, T]$ corresponding to $u$ is called admissible if $u$ is, in which case we shall refer to $(u, X)$ as an admissible pair. We note that we allow for $U$ to be a set-valued function of $t$ and $X$.

Suppose we are given a performance criterion $J(u)$ of the form

$$
J(u)=\mathrm{E}\left[\int_{0}^{T} f(t, X(t), u(t)) \mathrm{d} A(t)+g(X(T))\right]
$$

where $g$ and $f$ are continuous. We shall agree that any $u$ that violates

$$
\mathrm{E}\left[\int_{0}^{T} f^{-}(t, X(t), u(t)) \mathrm{d} A(t)+g^{-}(X(T))\right]<\infty
$$

is not admissible, where $y^{-}=\max (0,-y)$. An admissible $u^{*}$ maximizing $J$ is called an optimal control, and if we write $X^{*}=X^{\left(u^{*}\right)}(t)$, we call $\left(u^{*}, X^{*}\right)$ an optimal pair. 
Denote by $\mathrm{R}_{\lambda}$ the set of measurable functions $r: \Xi \rightarrow \mathbf{R}^{d}$ so that the integral in (6) below converges. Introduce the Hamiltonian $H:[0, T] \times \mathbf{R}^{d} \times U \times \mathbf{R}^{d} \times \mathbf{R}^{n \times d} \times \mathbf{R}_{\lambda}$ :

$$
\begin{aligned}
H(t, x, v, p, q, r(\cdot))= & f(t, x, v)+p \cdot b(t, x, v)+\sum_{i, j=1}^{n} \sigma_{j}(t, x, v) \cdot q_{i} \beta_{i j} \\
& +\int[r(z) \cdot \eta(t, x, v, z)+(p \cdot \eta(t, x, v, z)+r(z) \cdot x)(1-\chi)] \lambda(\mathrm{d} z)
\end{aligned}
$$

where $q=\left(q_{1}, \ldots, q_{n}\right)$ and « $\cdot \gg$ denotes the usual Euclidean inner product. Corresponding to the admissible pair $(u, X)$, the adjoint equation in the unknown adapted processes $p(t)$, $q(t)=\left(q_{1}(t), \ldots, q_{n}(t)\right)$ and $r(t, z)$ is the backward stochastic differential equation

$\mathrm{d} p(t)=-\frac{\partial}{\partial x} H(t, X(t), u(t), p(t), q(t), r(t, \cdot)) \mathrm{d} A(t)+\sum_{i=1}^{n} q_{i}(t)^{\top} \mathrm{d} Z_{i}(t)+\int r\left(t^{-}, z\right) \bar{M}(\mathrm{~d} t, \mathrm{~d} z)$

$p(T)=\nabla g(X(T))$

with the convention that $\partial H / \partial x$ and $\nabla g$ are allowed to be taken as supergradients if necessary. With that convention, we have the following sufficient condition for optimality:

\section{THEOREM 1.}

Let $\left(u^{*}, X^{*}\right)$ be an admissible pair and suppose there exists a solution $\hat{p}=\hat{p}(t), \hat{q}=\hat{q}(t)$, $\hat{r}=\hat{r}(t, z)$ of the corresponding adjoint equation (7), and such that

$$
\mathrm{E}\left[\int_{0}^{T}\left\{\sum_{i, j=1}^{n} \hat{q}_{i}(t)^{\top} \hat{q}_{j}(t) \beta_{i j}+\int \hat{r}(t, z)^{\top} \hat{r}(t, z) \chi(z) \lambda(\mathrm{d} z)\right\} \mathrm{d} A(t)\right]<\infty
$$

Moreover, suppose that

$$
\hat{H}(x):=\sup _{v \in U} H(t, x, v, \hat{p}(t), \hat{q}(t), \hat{r}(t, \cdot))
$$

exists and is concave, that $g$ is concave and that almost surely,

$$
\hat{H}\left(X^{*}(t)\right)=H\left(t, X^{*}(t), u^{*}(t), \hat{p}(t), \hat{q}(t), \hat{r}(t, \cdot)\right)
$$

on $[0, T]$ (i.e, the supremum is attained, and by $u^{*}$.) If also we have $J(u)<\infty$ for all admissible $u \neq u^{*}$, then $\left(u^{*}, X^{*}\right)$ is an optimal pair.

Proof. To simplify notation in the following, we shall let the «* $^{*}$ superscript signify evaluation at $\left(u^{*}(t), X^{*}\left(t^{-}\right)\right)$; elsewhere, we evaluate at $\left(u(t), X\left(t^{-}\right)\right)$while in both cases, we evaluate at $t^{-}, \hat{p}\left(t^{-}\right), \hat{q}\left(t^{-}\right)$and $\hat{r}\left(t^{-}, \cdot\right)$. Let $(u, X)$ be an arbitrary admissible pair and consider $J^{*}-J$. First, note that by concavity of $g$, we have for fixed $y$ that $g(y)-g(x) \geq \nabla g(y) \cdot(y-x)$ for all $x$ if $\nabla g$ is a supergradient. Therefore,

$$
g\left(X^{*}(T)\right)-g(X(T)) \geq \hat{p}(T) \cdot\left(X^{*}(T)-X(T)\right) \text { for a.a. } \omega \text {. }
$$


Using (6) and (11), we have:

$$
\begin{aligned}
& J\left(u^{*}\right)-J(u)=\mathrm{E}\left[\int_{0}^{T}\left(f^{*}-f\right) \mathrm{d} A(t)+g\left(X^{*}(T)\right)-g(X(T))\right] \\
& \geq \mathrm{E}\left[\int _ { 0 } ^ { T } \left\{\left(H^{*}-H\right)-\hat{p} \cdot\left(b^{*}-b\right)-\sum_{i, j=1}^{n}\left(\sigma_{j}^{*}-\sigma_{j}\right) \cdot \hat{q}_{i} \beta_{i j}\right.\right. \\
& \left.\quad-\int\left[\hat{r} \cdot\left(\eta^{*}-\eta\right)+\left(\hat{p} \cdot\left(\eta^{*}-\eta\right)+\hat{r} \cdot\left(X^{*}-X\right)\right)(1-\chi)\right] \lambda(\mathrm{d} z)\right\} \mathrm{d} A \\
& \left.\quad+\hat{p}(T) \cdot\left(X^{*}(T)-X(T)\right)\right] .
\end{aligned}
$$

Now we use the product rule for differentiation on the latter term. Recall the differential equations (2) and (7); by (3) and (8) (and polarization), the integrals with respect to the local martingales vanish when we take expectation:

$$
\begin{aligned}
& \mathrm{E}\left[\hat{p}(T) \cdot\left(X^{*}(T)-X(T)\right)\right]=\mathrm{E}\left[\int _ { 0 } ^ { T } \left\{\hat{p} \cdot\left(b^{*}-b\right)-\left(X^{*}-X\right) \cdot\left(\frac{\partial H}{\partial x}\right)^{*}+\sum_{i, j=1}^{n}\left(\sigma_{j}^{*}-\sigma_{j}\right) \cdot \hat{q}_{i} \beta_{i j}\right.\right. \\
& \left.\left.+\int\left[\left((\hat{p}+\hat{r}) \cdot\left(X^{*}-X+\eta^{*}-\eta\right)-\hat{p} \cdot\left(X^{*}-X\right)\right)(1-\chi)+\hat{r} \cdot\left(\eta^{*}-\eta\right) \chi\right] \lambda(\mathrm{d} z)\right\} \mathrm{d} A\right]
\end{aligned}
$$

Insert this into (12) and cancel terms, and we are left with

$$
J\left(u^{*}\right)-J(u) \geq \mathrm{E}\left[\int_{0}^{T}\left\{\left(H^{*}-H\right)-\left(X^{*}-X\right) \cdot\left(\frac{\partial H}{\partial x}\right)^{*}\right\} \mathrm{d} A\right] .
$$

Now by a convex analysis argument, optimality follows as in the deterministic case, cf. [13, p. 108]: recall that the «» denotes the pointwise maximization wrt. the $u$ variable, i.e. $H \leq \hat{H}$ for any fixed $u$. By assumption, that maximization is already performed when evaluating at

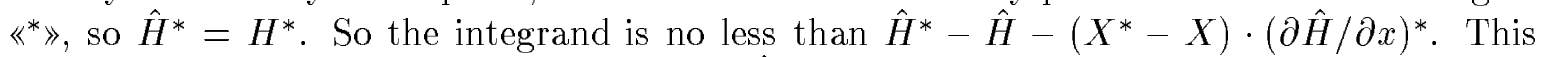
is convex in $X$ by the assumed concavity of $\hat{H}$, and is zero at the stationary point $X=X^{*}$. Thus the integrand is positive, and therefore $J\left(u^{*}\right)-J(u) \geq 0$ for all admissible $u$, hence (the admissible) $u^{*}$ is optimal.

\section{Ramifications}

\subsection{Explicit $\omega$-dependence}

It is straightforward to generalize the setting allowing for $\beta$ to be predictable, and for explicit $\omega$-dependence in the coefficients $b, \sigma_{i}, \eta$ and $\lambda$ of the differential equation (2) and also in $f$ and $U$ as long as for fixed $x$ and $u, b(\cdot, x, u, \cdot):[0, T] \times \Omega \rightarrow \mathbf{R}^{d}$ is predictable, and similarly for the other functions. The author chose not to include this $\omega$-dependence in the previous section 
for mainly three reasons; one, we would get an $\omega$-dependence in the adjoint equation (7), which could cause confusion as the term «backward stochastic differential equation» usually does not refer to this case; second, a theory for such equations is to the author's knowledge not developed; third, our use of the Itô formula does not à priori admit $\omega$-dependence in the terminal value function $g$; though there exists a generalization to allow for explicit $\omega$ dependence, see [11, Theorem V.18], this is essentially to a situation where $g$ can be written as a $\mathrm{d} A$ integral, which may be included in $f$. We note, though, that we may in principle let $T$ be a stopping time, which may however destroy the applicability. As an example, we mention the case where $T$ is a Poisson jump independent of everything else; then we have not much hope for $(7 \mathrm{~b})$ to hold (although this particular case may easily be reduced to a problem with $T$ nonrandom.)

\section{$2.2 \quad$ Singular controls}

In the Brownian case, the term «singular stochastic control» refers to control processes which are singular to the time Lebesgue measure $\mathrm{d} t$, which in our setting generalizes to $[Z, Z]$. We may adapt to this setting by Lebesgue decomposing $A=\sum_{i \in I} A_{i}$ and performing similar decompositions in the coefficients and $f$-and also $H$. If the index set $I$ is infinite, we need arguments to ensure convergence; however, a typical singular stochastic control problem is posed as to finding a finite number of local time-esque reflections, which may be translated into finding these finitely many $A_{i}$ 's.

\subsection{Terminal-/transversality conditions}

The extension to almost sure $\omega$-wise terminal-/transversality conditions is straightforward; as in the deterministic case, transversality ensures (11). Furthermore, we note that we do not need the almost sure inequality (11), as long as

$$
\mathrm{E}\left[g\left(X^{*}(T)\right)-g(X(T))\right] \geq \mathrm{E}\left[\hat{p}(T) \cdot\left(X^{*}(T)-X(T)\right)\right]
$$

which is the transversality condition which will grant the inequality in (12). The closing argument of the below example is a guise of this generalization.

\subsection{Uniqueness}

Strict concavity of $H$ or $g$ - or strict inequality in (15) - is sufficient to grant that $u^{*}$ is uniquely optimal, because we have assumed that $J(u)<\infty$ except possibly for $u^{*}$. Strict concavity may be weakened if $X$ will with positive probability spend positive time on a set on which strict concavity holds.

\subsection{Catching up or overtaking with possibly infinite horizon}

Let us modify the setup. Consider for each $T$

$$
J_{T}(u)=\mathrm{E}\left[\int_{0}^{T} f(t, X(t), u(t)) \mathrm{d} A(t)\right]
$$

(i.e. $g=0$ ) and suppose that $J_{T} \in \mathbf{R}$ for all $T<\bar{T}$, and all admissible $u$. Now $\bar{T}$ (finite or infinite) is our horizon; à priori, we would want to maximize $J_{\bar{T}}$, but this criterion may be too 
weak if $J_{\bar{T}}= \pm \infty$, which is now allowed. We can modify (7b) to get sufficient transversality conditions for catching up or overtaking criteria:

\section{THEOREM 2.}

a) «Catching up» type criteria:

Assume that the hypothesis of Theorem 1 holds, except that in place of $(7 \mathrm{~b})$ we assume that for all sufficiently small $\epsilon>0, \hat{p}$ satisfies

$$
\epsilon \geq \mathrm{E}\left[\hat{p}\left(\tau_{n}\right) \cdot\left(X^{*}\left(\tau_{n}\right)-X\left(\tau_{n}\right)\right)\right]
$$

for a sequence $\left\{\tau_{n}\right\}$ of stopping times $\tau_{n} \nearrow \bar{T}$. Then $u^{*}$ sporadically catches up with $u$ in the sense that

$$
J_{\tau_{n}}\left(u^{*}\right) \geq J_{\tau_{n}}(u)-\epsilon .
$$

If (16) holds for all large enough deterministic $\tau_{n}$, and all admissible $u$, then $u^{*}$ catches up with any $u$ in the usual sense.

b) «Overtaking» type criteria:

If we modify i) above by putting $\epsilon=0$ in (16), then $u^{*}$ will (sporadically) overtake $u$ in the sense analogous to (17). If also (16) holds with strict inequality for $\epsilon=0$ for any admissible $u$, we have the uniqueness property that $u^{*}$ will strictly overtake any admissible $u \neq u^{*}$ in the sense analogous to (17).

Proof. For each $\tau=\tau_{n}$ we get a modified version of (12):

$$
\begin{aligned}
J_{\tau}\left(u^{*}\right)-J_{\tau}(u)=\mathrm{E}\left[\int_{0}^{\tau}\left(f^{*}-f\right) \mathrm{d} A(t)\right] \\
\geq \mathrm{E}\left[\int _ { 0 } ^ { \tau } \left\{\left(H^{*}-H\right)-\hat{p} \cdot\left(b^{*}-b\right)-\sum_{i, j=1}^{n}\left(\sigma_{j}^{*}-\sigma_{j}\right) \cdot \hat{q}_{i} \beta_{i j}\right.\right. \\
\left.\quad-\int\left[\hat{r} \cdot\left(\eta^{*}-\eta\right)+\left(\hat{p} \cdot\left(\eta^{*}-\eta\right)+\hat{r} \cdot\left(X^{*}-X\right)\right)(1-\chi)\right] \lambda(\mathrm{d} z)\right\} \mathrm{d} A \\
\left.+\hat{p}(\tau) \cdot\left(X^{*}(\tau)-X(\tau)\right)\right]-\epsilon .
\end{aligned}
$$

To apply similar arguments as in the proof of Theorem 1, we merely need to note that the Itô formula admits $\tau$ random.

\section{An application to optimal portfolio selection}

It is typically not easy to solve backward stochastic differential equations driven by a general semimartingale, but in special cases we may find a solution more or less by guess. We shall give an example with a portfolio choice problem in finance. Assume we have a market with two investment opportunities, a safe «money market» $S_{0}$ and a risky «stock» $S$ driven by a real-valued martingale $Z$. They are assumed to evolve according to the geometric processes

$$
\begin{aligned}
& \mathrm{d} S_{0}(t)=r S_{0}(t) \mathrm{d} A(t) \\
& \mathrm{d} S_{1}(t)=S_{1}(t)(\mu \mathrm{d} A(t)+\sigma \mathrm{d} Z(t)) .
\end{aligned}
$$


where $A=[Z, Z]$ and where $\sigma>0, \mu$ and $r$ are constants. By discounting, we can and will assume that $r=0$. From these assets, we form a self-financing portfolio, so that wealth will follow

$$
\mathrm{d} X(t)=u(t) X(t)(\mu \mathrm{d} A(t)+\sigma \mathrm{d} Z(t))
$$

where our control $u(t)$ is the fraction of the wealth invested in the stock at time $t$. We assume that the agent wants to maximize expected terminal utility of constant relative risk aversion type, i.e. that $f=0$ while $g^{\prime}(x)=x^{\gamma-1}$ for some constant $\gamma<1$. The Hamiltonian $H$ then takes the form

$$
(p \mu+\sigma q) u X
$$

This is linear in $v$, so in order to have a finite solution we try $q=-p \mu / \sigma$ and get the following backward equation

$$
\mathrm{d} p(t)=-p(t) \frac{\mu}{\sigma} \mathrm{d} Z, \quad p(T)=(X(T))^{\gamma-1}
$$

Solving for $p(t)$ in terms of the initial value $p(0)$, we get the Doleans exponential

$$
p(t)=p(0) \exp \left\{-\frac{\mu}{\sigma} Z(t)-\frac{1}{2}\left(\frac{\mu}{\sigma}\right)^{2} A(t)\right\} .
$$

Inspired by the geometric Brownian case, we try $u(t)$ constant, so that $X$ is a geometric process and

$$
X(T)=X(0) \exp \left\{u \sigma Z(T)+\left(u \mu-\frac{1}{2} u^{2} \sigma^{2}\right) A(T)\right\} .
$$

Let us match terminal values:

$$
\ln p(0)-\frac{\mu}{\sigma} Z(T)-\frac{1}{2}\left(\frac{\mu}{\sigma}\right)^{2} A(T)=(\gamma-1)\left[\ln X(0)+u \sigma Z(T)+\left(u \mu-\frac{1}{2} u^{2} \sigma^{2}\right) A(T)\right]
$$

so our candidate for the optimal strategy will be determined by the $Z(T)$ coefficient:

$$
u(t)=\frac{\mu}{\sigma^{2}(1-\gamma)}
$$

i.e. just as in the case where $Z$ is standard Brownian motion. But then we face the following problem: this $u$ value will yield

$$
\ln p(0)=\left[(\gamma-1) \ln X(0)-\frac{1}{2} \frac{\gamma}{1-\gamma}\left(\frac{\mu}{\sigma}\right)^{2}\right] A(T)
$$

which is not $\mathcal{F}_{0}$-measurable unless the particular value $A(T)$ is non-random; in that case, however, the generalization from the Brownian case (more or less) vanishes, as a continuous martingale with bracket $A$ (essentially) is a Brownian motion living on $A$ (cf. e.g. [9, Section 3.4.B]), and the problem is no more than a time change of the Brownian case. However, we note that the strategy (20) does not depend on the value of $A(T)$. Therefore, we have the following:

\section{THEOREM 3.}

Let for each each $t \mathcal{G}_{t}$ be generated by $\mathcal{F}_{t}$ and the single random variable $A(T)$. Suppose that with respect to the filtration $\left\{\mathcal{G}_{t}\right\}$ we still have $Z$ a martingale with bracket $[Z, Z]=A$. Then the above example has optimal control given by $(20)$.

Proof. The problem is solved under $\mathcal{G}_{t}$, with the optimal control being $\mathcal{F}_{t}$-adapted.

As we now allow for $A(T)$ nondeterministic, this is a generalization of the Brownian setup. 


\section{References}

[1] F. Antonelli, Backward-forward stochastic differential equations, Ann. Appl. Probab. 3 (1993), 777-793.

[2] A. Bensoussan, Maximum principle and dynamic programming approaches of the optimal control of partially observed diffusions, Stochastics, 9 (1983), 169-222.

[3] J.-M. Bismut, Conjugate convex functions in optimal stochastic control, J. Math. Anal. and Appl. 44 (1973), 384-401.

[4] A. Cadenillas and U. G. Haussmann, The stochastic maximum principle for a singular control problem, Stochastics and Stochastics Reports, 49 (1994), 211-237.

[5] N. C. Framstad, B. Øksendal and A. Sulem, A sufficient stochastic maximum principle for optimal control of jump diffusions and applications to finance, Preprint Pure Mathematics 22/2001, University of Oslo.

[6] U. Haussmann, A Stochastic Maximum Principle for Optimal Control of Diffusions, Longman Scientific and Technical (1986).

[7] Y. Kabanov, On the Pontryagin maximum principle for SDEs with a Poisson-type driving noise. In Kabanov et al. (eds): Statistics and Control of Stochastic Processes. World Scientific (1997), 173-190.

[8] H. J. Kushner, Necessary conditions for continuous parameter stochastic optimization problems, SIAM J. Control 10 (1972), 550-565.

[9] I. Karatzas and S. Shreve, Brownian motion and stochastic calculus, second edition. Springer-Verlag (1991).

[10] S. Peng, A general stochastic maximum principle for optimal control problems, SIAM J. Control \& Optim. 28 (1990), 966-979.

[11] P. Protter, Stochastic integration and differential equations. A new approach, SpringerVerlag (1990).

[12] A. Seierstand and K. Sydsæter, Sufficient conditions in optimal control theory, Internat. Econom. Rev. 18 (1977), 367-391.

[13] A. Seierstand and K. Sydsæter, Optimal Control Theory with Economic Applications, North-Holland (1987).

[14] S. J. Tang and X. J. Li, Necessary conditions for optimal control of stochastic systems with random jumps, SIAM J. Control Optim. 32 (1994), 1447-1475. 